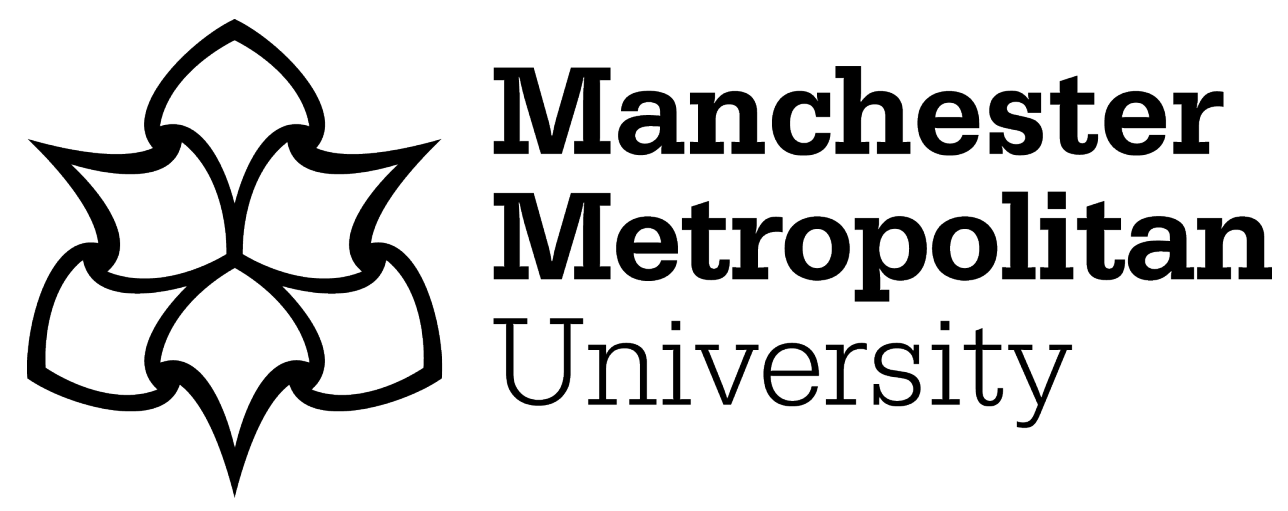

Anoh, K, Adebisi, B, Rabie, KM and Gacanin, H (2018) Optimization of Impulsive Noise Mitigation Scheme for PAPR Reduced OFDM Signals Over Powerline Channels. In: IEEE 87th Vehicular Technology Conference (VTC Spring 2018), 03 June 2018 - 06 June 2018, Porto, Portugal. (In Press)

Downloaded from: https://e-space.mmu.ac.uk/620769/

Version: Accepted Version

Publisher: IEEE

DOI: https://doi.org/10.1109/VTCSpring.2018.8417536

Please cite the published version 


\title{
Optimization of Impulsive Noise Mitigation Scheme for PAPR Reduced OFDM Signals Over Powerline Channels
}

\author{
Kelvin Anoh*, Bamidele Adebisi*, Khaled M. Rabie* and Haris Gacanin ${ }^{\dagger}$ \\ * Manchester Metropolitan University, United Kingdom \\ ${ }^{\dagger}$ Nokia Bell Labs, Belgium \\ [k.anoh, b.adebisi, k.rabie]@mmu.ac.uk, haris.gacanin@ nokia-bell-labs.com
}

\begin{abstract}
The IEEE 1901 powerline standard can be deployed using orthogonal frequency division multiplexing (OFDM) since it is robust over impulsive channels. However, the powerline channel picks up impulsive interference that the conventional OFDM driver cannot combat. Since the probability density function (PDF) of OFDM amplitudes follow the Rayleigh distribution, it becomes difficult to correctly predict the existence of impulsive noise (IN) in powerline systems. In this study, we use companding transforms to convert the PDF of the conventional OFDM system to a uniform distribution which avails the identification and mitigation of IN. Results show significant improvement in the output signal-to-noise ratio (SNR) when nonlinear optimization search is applied. We also show that the conventional PDF leads to false IN detection which diminishes the output SNR when nonlinear memoryless mitigation scheme such as clipping or blanking is applied. Thus, companding OFDM signals before transmission helps to correctly predict the optimal blanking or clipping threshold which in turn improves the output SNR performance.
\end{abstract}

Index Terms-Powerline, OFDM, Impulsive Noise, Uniform Distribution, Companding, Optimization.

\section{INTRODUCTION}

At homes, wireless signals can be severely attenuated by thick solid objects limiting signal penetration into certain areas of a building. This can protract broadband services, home automation, monitoring, security and comfort, etc. Consequently, powerline communication (PLC) systems such as the IEEE 1901 may be used to transmit data at homes and microgrids over electric power cables [1], [2]. An interference from different home appliances (i.e., disturbers) influences the performance of PLC signals over the powerline channels. For example, such disturbers induce non-Gaussian noise into the powerline channel that corrupt the signal amplitude and consequently makes it unrecoverable.

The IEEE 1901 standard uses orthogonal frequency division multiplexing (OFDM) for physical layer signaling over powerlines which may operate using wavelets or FFT [1], [3]-[5]. OFDM is a robust scheme which combats impulse responses of the channels by deploying cyclic prefix that is at least as long as the worst impulse response. OFDM achieves this by partitioning a wide-band into many narrow-bands subcarriers such that symbol time is increased. Unfortunately, the amplitude distribution of OFDM signals with a large number of subcarriers follows a Rayleigh instead of uniform distribution. This implies that a percentage of OFDM signals have amplitudes distributed above the mean (i.e., assuming that the transmit signal is normalized) amplitude beclouds the identification of impulsive noise (IN) in the powerline system. Practically, these small fractions of amplitudes distributed beyond the mean amplitude lead to high peak-to-average power ratio (PAPR). In other words, they lead to lower efficiency of high power amplifier (HPA) with operation close to the saturation region. More importantly, it becomes difficult, if not impossible, to identify and mitigate the IN of the powerline channel due to the uneven distribution of these amplitudes.

PAPR can be reduced by applying clipping or companding after OFDM modulation at the transmitter [6]-[8]. This leads to converting the distribution of signal amplitude from Rayleigh to a uniform distribution. The concept of improving IN mitigation by reducing PAPR at transmitter was first introduced in [9]. In addition, OFDM systems dispensing with a flat envelope was later proposed to reduce PAPR and avail amplitude distributions that can help mitigate IN [10], however the scheme imposes computational burden on the system. While the clipping or companding PAPR techniques are performed after OFDM modulation at the transmitter [6], [7], the nonlinear blanking or clipping to mitigate IN are performed at the receiver [11] before OFDM demodulation. Our study is motivated from studying companding transforms which have the ability to significantly reduce PAPR [6], [8], [12]-[14]. Thus, we exploit, in addition to reducing the PAPR, the companding transform for IN mitigation in powerline transmission of OFDM modulated signals. Some studies are done in [15], however, the work was limited to only $\mu$ law based companding transform without comparison with conventional OFDM performance.

In this study, we present a comprehensive system analysis discussing identification and mitigation of IN in PLC. We present different PDF models to approximate the Rayleigh distributed signal envelope of the conventional OFDM with a uniformly distributed signal envelope. In addition, we also present an optimization approach to search for amplitudes where signals can be clipped or blanked to achieve optimal system performance. The performance of five different companding transforms with detailed examination of their capabilities in mitigating IN is discussed in terms of output signalto-noise ratio (SNR) at the receiver, the PDF distributions 
of the signal amplitudes, optimization of model parameters and the companding transform performances. Furthermore, we applied the optimization to the search of optimal amplitudes for which the signals can be clipped or blanked to achieve the optimal SNR. The PDFs that characterize these companding transforms are hyperbolic arcsine companding (HASC) [16], exponential companding (EC) [17], $\mu$-law companding (MC) [6], log-based modified MC (LMC) [14] and the error-function companding (ERFC) [13] transforms. We find that among the studied PDFs, the ERFC transform outperforms all others, while the exponential transform is the least performing. The strength of the ERFC transform has been derived from the fact that it follows a uniform distribution better than others and thus leverages the easy identification and removal of the IN in the system.

\section{SySTEM MOdEL}

In the system mdoel, we consider an OFDM-driven powerline system with IN. As an example, consider a frequency domain data symbol $X=\left[X_{0}, X_{1}, X_{2}, \cdots, X_{N-1}\right]$ which can be transformed into its time domain component as

$x(n)=\frac{1}{\sqrt{N}} \sum_{k=0}^{N-1} X(k) \exp \left(j 2 \pi \frac{n k}{N}\right) \forall n=1,2, \cdots, N-1$

From (1), the resulting time domain OFDM signal can be decomposed into real $\left(x_{r}\right)$ and imaginary $\left(x_{i}\right)$ parts. Then, from the knowledge of central limit theorem, $x_{r}$ and $x_{i}$ are identically and independently distributed Gaussian random variables. This implies that for $x \sim \mathcal{N}\left(\mu_{x}, \sigma_{x}^{2}\right)$ the PDF of these $x_{r}$ and $x_{i}$ follows a Rayleigh distribution which can be expressed as

$$
f_{|x|}\left(x ; \mu_{x}, \sigma_{x}\right)=\frac{1}{\sqrt{2 \pi \sigma_{x}^{2}}} \exp \left(-\frac{1}{2}\left(\frac{x_{0}-\mu_{x}}{\sigma_{x}}\right)^{2}\right)
$$

where $\mu_{x}=\mathbb{E}\{x(n)\}, \mathbb{E}\{\cdot\}$ is the expected mean operator, $\sigma_{x}$ is the standard deviation, and $\sigma_{x}^{2}$ is the variance of $x(n)$. In (2), $x_{0}$ is the discrete envelope of $x(n)$ and can be depicted in Fig. 1 as having a Rayleigh distribution. Now, since the discrete envelope of $\boldsymbol{x}(n)$ - the amplitudes - follows a Rayleigh distribution, then majority of the signals have amplitudes distributed around the $\mu_{x}$ while a few others are distributed to the left-hand and right-hand sides of the distribution. Two problems are derived from these characteristics of the distribution; the high amplitude that appear at the right-hand side leads to high PAPR in the conventional OFDM and secondly the general non-uniform distribution of the amplitudes toughens the identification, isolation and mitigation of IN in powerline communication systems - the results are, respectively, high power consumption by HPAs and the other is poor performance in terms of the output power at the receiver.

When $x(n)$ is normally distributed, then $\mu_{x}=0$. On the other hand, the cumulative density function (CDF) of the Gaussian distributed variable $x(n)$ is the integration of the PDF and can be expressed in closed form as

$$
C_{x}\left(x ; \mu_{x}, \sigma_{x}\right)=\frac{1}{2}\left(1+\operatorname{erf}\left(\frac{1}{\sqrt{2}}\left(\frac{x_{0}-\mu_{x}}{\sigma_{x}^{2}}\right)\right)\right)
$$

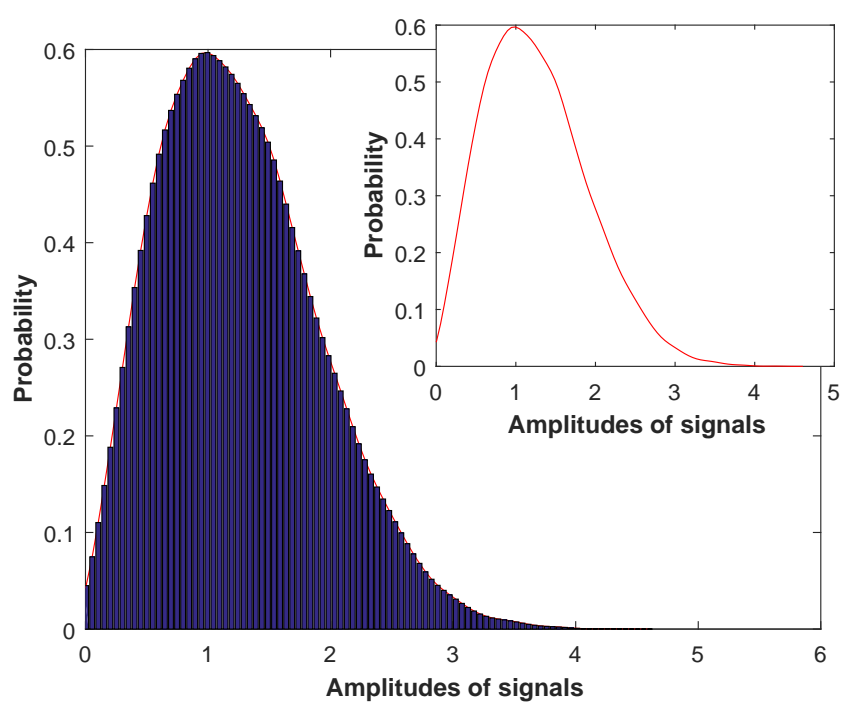

Figure 1. PDF distribution of OFDM signal demonstrating the amplitude distribution which influences its behaviour over powerline channel

where $\operatorname{erf}(\cdot)$ is the error function. Our goal therefore is to approximate the non-uniform OFDM amplitude distribution in such a way that the amplitudes become uniformly distributed so as to enhance the identification and mitigation of IN in powerline systems. Generally, the PAPR mitigation techniques that apply after the OFDM modulation can be either clipping or companding. Both are simple to implement but the companding transform excels in BER performance due to slight amplitude distortions impacted on the signals. Earlier, a study in [15] pursued this idea of using companding transform to mitigate IN in powerline systems but was limited to only one model of companding transform.

\section{Approximation of Signal EnVelope DISTRIBUTION}

Now, recall the time-domain OFDM signals in (1), it can be passed through a companding transform to change its PDF to a uniform distribution as follows [12], [13]

$$
\mathcal{F}(\boldsymbol{x}(n))=C_{x_{c}}^{-1}\left(C_{x}(x(n))\right)
$$

which can be rewritten as

$$
C_{x_{c}}(\mathcal{F}(\boldsymbol{x}(n)))=C_{x}(x(n))
$$

where $C_{x}(\cdot)$ is the $\mathrm{CDF}$ of the uncompanded signals, $C_{x_{c}}(\cdot)$ and $C_{x_{c}}^{-1}(\cdot)$ is the CDF and inverse CDF of the companded signal respectively. $\mathcal{F}(\boldsymbol{x}(n))$ is the resulting companding transform that converts the PDF of conventional OFDM to a uniform distribution. Since $\left|x_{c}(n)\right|$ tends to the desired uniform distribution, then the CDF is written as [13]

$$
C_{x_{c}(n)}\left(x_{c}\right)=\frac{x_{c}}{2 A}+\frac{1}{2}, 0 \leq x_{c} \leq A .
$$

By combining (3), (4) and (6), the companding transform can be expressed as

$$
\mathcal{F}_{1}(\boldsymbol{x}(n))=\operatorname{sgn}(x) \cdot A_{1} \cdot \operatorname{erf}\left(\frac{|x|}{\sqrt{2 \sigma_{x}^{2}}}\right), 0 \leq x \leq 1
$$


where $A_{1}=\sqrt{3 \sigma_{x}^{2}}$. Our target is however to achieve $[0, A]$, where $0<|\mathcal{F}(\boldsymbol{x}(n))| \leq 1$. Meanwhile, the foremost PAPR companding scheme uses the well-known $\mu$-law [6]

$$
\mathcal{F}_{2}(\boldsymbol{x}(n))=A_{2} \operatorname{sgn}(x(n)) \frac{\ln \left[1+\mu\left|\frac{x(n)}{A_{2}}\right|\right]}{\ln (1+\mu)}
$$

where $A_{2}$ is a normalization parameter confined within $0 \leq$ $\left|\frac{x(n)}{A_{2}}\right| \leq 1$. The problem with (8) is that it expands the amplitudes of lower energy signals without compressing the larger ones. Thus, in [14], (8) was modified as

$$
\mathcal{F}_{3}(x(n))=\operatorname{sgn}(x)\left(\alpha_{3} \times \ln \left[1+\mu\left|\frac{x(n)}{A_{3}}\right|\right]\right)^{\frac{1}{2 a_{3}}}
$$

where

$$
\alpha_{4}=\left(u / \mathbb{E}\left\{\sqrt[a_{3}]{\left(\log \left(1+\mu \frac{|x|}{A_{3}}\right)\right)^{b_{3}}}\right\}\right)
$$

where $u=\mathbb{E}\left\{|x|^{2}\right\}$. Based on the hyperbolic arcsine function, $\sinh ^{-1}(x)=\log \left(x+\sqrt{x^{2}+1}\right)$, [16] suggested a different companding transform of the form

$$
\mathcal{F}_{4}(x(n))=\beta_{4} \times \begin{cases}\operatorname{sgn}(x) \times \sinh ^{-1}(K|x|), & |x| \leq c A_{4} \\ \operatorname{sgn}(x) \times \sinh ^{-1}\left(K c A_{4}\right), & |x|>c A_{4}\end{cases}
$$

where $c$ and $K$ are the flexing-point determining parameters associated with $A_{4} . \beta_{4}$ is the parameter that normalizes the output power of the companded signal to be similar to that of the input signal. In [17], an exponential companding transform that can approximate the PDF of the conventional OFDM signal to a uniform distribution was proposed such as

$$
\begin{aligned}
\mathcal{F}_{5}(x(n)) & =\operatorname{sgn}(x) \sqrt[d_{5}]{\beta_{5}\left[1-\exp \left(-\frac{x^{2}}{\sigma_{x}^{2}}\right)\right]} \\
\beta_{5} & =\left(u / \mathbb{E}\left\{\sqrt[d_{5}]{\left[1-\exp \left(-\frac{x^{2}}{\sigma_{x}^{2}}\right)\right]^{2}}\right\}\right)^{\frac{d_{5}}{2}}
\end{aligned}
$$

where $d_{5}>0$ and in general, $\operatorname{sgn}(x)=\frac{x(n)}{|x(n)|}$ is the phase. In Fig. 4, we analyze the companding performances of these transforms in terms of amplitude compression and expansion respectively of input signals. MC scheme expands the amplitudes of lower energy signals without impacts on the high energy signals. This will increase the output SNR. It is followed by LMC although LMC expands the amplitude of lower energy signals and also compresses the amplitudes of higher energy signals; this is similarly true for ERFC. On the other hand, HASC does not impact the amplitudes of lower energy signals, however it compresses the amplitude of high energy signals. Lastly, the EC compresses the amplitudes of high amplitude signals and expands the low energy ones; this will greatly improve the distribution towards the desired uniform distribution. Meanwhile, it is worthy to mention that companding involves compressing the amplitudes of the amplitudes of large amplitude (high energy) signals and expanding low amplitude (low energy) signals. While some companding

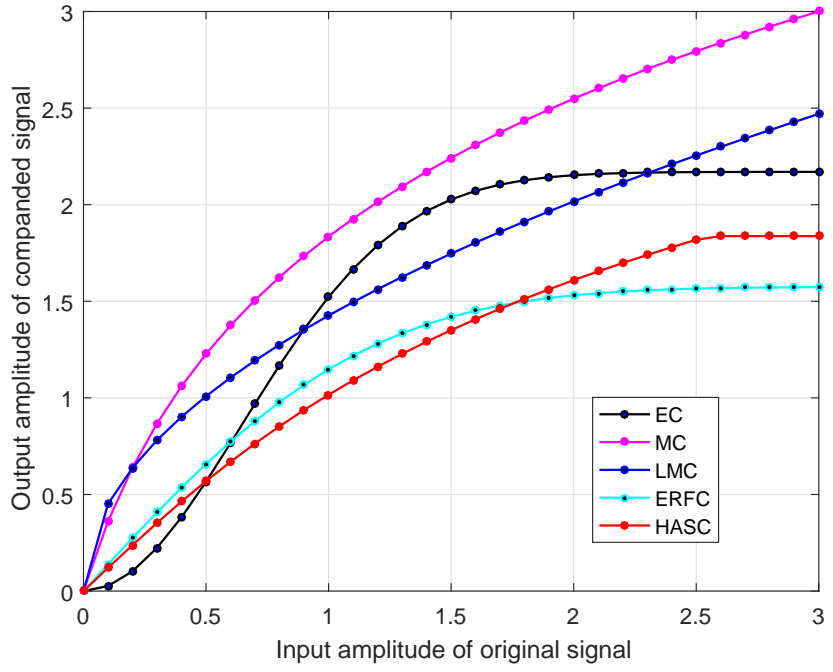

Figure 2. Comparisons of different companding transforms

transforms achieve the former others achieve the latter only. Ideally, effective companding transforms achieve both characteristics simultaneously and their performances in availing IN mitigation lies on how much of uniform distribution that is achieved.

\section{A. Companded OFDM Signal Transmission over Powerline Channels with Impulsive Noise}

Our investigation is on the companded OFDM signal transmitted over powerline channels with IN. In other words, we consider digital signal transmission over a memoryless IN channel with characteristic Gaussian noise, $z_{w} \sim \mathcal{N}\left(0, \sigma_{w}^{2}\right)$ and IN $z_{i} \sim \mathcal{N}\left(0, \sigma_{i}^{2}\right)$ where $\sigma_{w}^{2}$ and $\sigma_{i}^{2}$ are the variances. Assuming that the total noise samples $z(n)=z_{w}(n)+z_{i}(n)$ are uncorrelated, then the distribution fits into the mixtureGaussian model and the PDF can be expressed as [11], [18]

$f_{z}\left(z ; \mu_{z}, \sigma_{z}\right)=\sum_{l=0}^{L=1} p_{l} \mathcal{N}\left(z_{0}(n) ; 0, \sigma_{z, l}^{2}\right) \forall n=1,2, \cdots, N-1$

where $\mathcal{N}\left(z_{0} ; 0, \sigma_{z, l}^{2}\right)=\frac{1}{\sigma_{z, l} \sqrt{2 \pi}} \exp \left(-\frac{1}{2}\left(\frac{z_{0}-\mu_{z}}{\sigma_{z, l}}\right)^{2}\right)$ is the Gaussian PDF of $z(n)$ with $z_{0}$ discrete envelope, zero-mean $\left(\mu_{z}=0\right)$, variance $\sigma_{z, l}^{2}$ and $p_{l}$ is the mixing probability of the $l^{t h}$ component. From (12), we can separate the mixing probability into $p_{0}=1-p$ and $p_{1}=p$, where $p$ is the probability of IN occurrence. Similarly, the variance can be separated into $\sigma_{z, 0}^{2}=\sigma_{w}^{2}$ and $\sigma_{z, 1}^{2}=\sigma_{w}^{2}+\sigma_{i}^{2}$. Meanwhile, the result of the companded signal can now be expressed as

$$
x_{c t}(n)=\mathcal{F}(\boldsymbol{x}(n)), \forall n=0,1, \cdots, N-1 .
$$

When OFDM signals are companded, the signal undergoes some nonlinear amplitude distortion and can be described from the Bussgang theory as [19]

$$
x_{c c}(n)=\mathcal{F}(\boldsymbol{x}(n))=\alpha_{n} x(n)+D_{n} \forall n=0,1, \cdots, N-1
$$


where $\alpha_{n}$ is the attenuation factor and $D_{n}$ is distortion noise. Thus, before transmission, we compensate the amplitude distorted signal as follows

$$
x_{c}(n)=\bar{\alpha}_{n} x_{c c}(n),
$$

where $\bar{\alpha}_{n}$ is the correlation coefficient of the distorted and original signal that minimizes the error in $\mathbb{E}\left[\left|\boldsymbol{x}(n)-\mathcal{R} \hat{\boldsymbol{x}}^{*}(n)\right|^{2}\right]$ after power amplification/reduction from companding which can be written as [20]

$$
\bar{\alpha}_{n}=\frac{\mathbb{E}\left[\boldsymbol{x}(n) \cdot x_{c}^{*}(n)\right]}{\mathbb{E}\left[\left|x_{c}(n)\right|^{2}\right]},
$$

where $(\cdot)^{*}$ represents complex conjugate operator. In general, therefore, the received signal at the destination PLC modem can be expressed as

$$
\begin{aligned}
\boldsymbol{r}(n) & =\boldsymbol{x}_{c}(n)+\boldsymbol{z}(n) \\
& =\mathcal{F}(\boldsymbol{x}(n))+\boldsymbol{z}_{w}(n)+\boldsymbol{z}_{i}(n) .
\end{aligned}
$$

where $z_{i}(n)$ represents the IN component. Although the OFDM symbol duration is are usually long which can mitigate the impulsive nature of the channel, this is only holds if the impulsive energy is moderate [11]. Since the IN amplitude is usually longer, some memoryless nonlinearities such as clipping and blanking are required before the OFDM demodulator.

\section{B. Clipping and Blanking Nonlinear Preprocessors}

We assume that the nonlinear mitigation schemes that are applied to reduce the IN effect corresponds to the signal sampled at Nyquist sampling rate. This implies that all distortion suffered by the signal are all within the in-band. For our system, the two nonlinear mitigation approaches are applied at the front-end of the OFDM system, in which all schemes are based on the ideal that for $\left|x_{c}(n)\right|>T$, then the signal is clipped or blanked. Considering clipping first, we express the output clipped signals as

$$
y(n)= \begin{cases}r(n) & |r(n)| \leq T_{c} \\ T_{c} \times \exp (\arg \{r(n)\}) & |r(n)|>T_{c}\end{cases}
$$

where $T_{c}$ is the clipping threshold. Similarly for blanking scheme, we express the output blanked signal as

$$
y(n)= \begin{cases}r(n) & |r(n)| \leq T_{b} \\ 0 & |r(n)|>T_{b}\end{cases}
$$

where $\arg \{\cdot\}$ estimates the angle and $T$ is the blanking threshold. At the output of the nonlinear memoryless preprocessors, the SNR can be found as [11]

$$
\begin{aligned}
S N R_{\text {out }} & =10 \log _{10}\left\{\frac{\mathbb{E}\left\{\mid K_{0} \mathcal{F}\left(\left.x(n)\right|^{2}\right\}\right.}{\mathbb{E}\left\{\mid y(n)-K_{0} \mathcal{F}\left(\left.x(n)\right|^{2}\right\}\right.}\right\} \\
& =10 \log _{10}\left\{\left(\frac{E_{\text {out }}}{2 K_{0}^{2}}-1\right)^{-1}\right\}
\end{aligned}
$$

where $E_{\text {out }}=\mathbb{E}\left\{|y(n)|^{2}\right\}$ is the output power of the nonlinearly mitigated signal, $K_{0}$ is a scaling factor. Indeed,

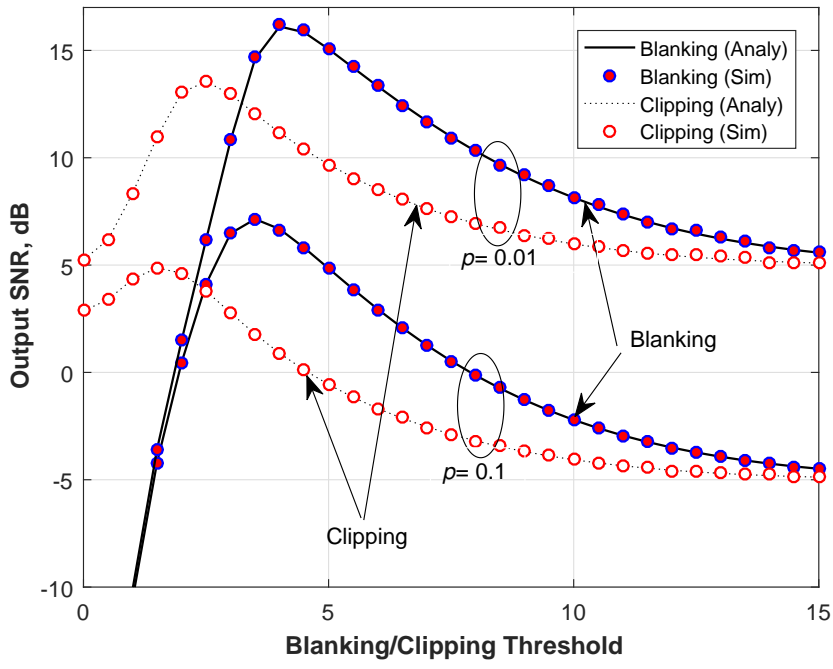

Figure 3. Comparisons of clipping and blanking performances in terms of the output SNR after nonlinear memoryless mitigation; SNR $=25 \mathrm{~dB}$ and $\mathrm{SINR}=$ $-15 \mathrm{~dB}, \mathrm{~N}=4096$

the in-band distortions induced by the clipping scheme thus diminishes the output SNR so that the blanking scheme outperforms the clipping scheme in Fig. 3. Consequently, we shall continue our study with the blanking scheme in the subsequent sections. Meanwhile, observe that the simulated and the analytical results perfectly agree for both at $p=0.01$ and $p=0.1$.

\section{Optimization of Preprocessing Parameters}

In this section, we present an optimization approach to finding the optimal blanking amplitude for mitigating impulsive that have been availed by companding OFDM signals before transmission. Since the IN occurrence cannot be predicted precisely, we maximize the performance of our system through some threshold selection process as in [15], [21] involving some model parameters such as

$$
\begin{gathered}
T_{\text {opt }}^{\text {blank }}=\underset{\substack{0 \leq T \leq A_{\max } \\
\text { subject to }}}{\arg \max } \gamma^{\text {blank }}(T, p, \mathrm{SINR}, \mathrm{SNR}) \\
A_{\max }=\arg \max _{0 \leq n \leq N-1}(|x(n)|)
\end{gathered}
$$

where SINR is the signal-to-interference plus noise ratio. Recall that our goal, for example, is to establish the best performing companding transform that can achieve most reduction of the IN in the powerline system, we apply optimization to finding the maximal received SNR and the optimal blanking threshold given the different companding transforms. Consequently, we modify (21) to include $\mathcal{F}_{m}(\boldsymbol{x}(n))$ as

$$
T_{\text {opt }}^{\text {blank }}=\underset{0 \leq T \leq A_{\max }}{\arg \max } \gamma^{\text {blank }}\left(T, \mathcal{F}_{m}(\boldsymbol{x}(n)), p, \mathrm{SINR}, \mathrm{SNR}\right)
$$

subject to

$$
\begin{aligned}
A_{\max } & =\arg \max _{0 \leq n \leq N-1}(|x(n)|) \\
\forall m & =1, \cdots, 5 .
\end{aligned}
$$


It can be observed that (22) involves one objective variable $\mathcal{F}_{m}(\boldsymbol{x}(n))$ which is considered one at a time. We define $\gamma^{\text {blank }}$, the output SNR after blanking nonlinear memoryless processing as

$$
\gamma^{\text {blank }}=\frac{\mathbb{E}\left\{K_{0}^{\text {blank }}\left|x_{c}(n)\right|^{2}\right\}}{\mathbb{E}\left\{|y(n)|^{2}-K_{0}^{\text {blank }}\left|x_{c}(n)\right|^{2}\right\}}
$$

where $K_{0}^{\text {blank }}$ is a scaling parameter. Meanwhile, $p_{0}$ and $p_{1}$ are dependent on $p$ which have chosen to be $p=\{0.1,0.01\}$.

\section{REsults AND Discussion}

From the ongoing discussion, our investigation involves OFDM signals that is companded to improve the detection and mitigation of IN. To realize this, we use $N=4096$ OFDM signal frame and modulated these data symbols using 16-QAM before passing them through an IFFT-block to obtain the time-domain signals represented in (1). After applying the companding transform, the resulting signals are transmitted over the IN channel with additive white Gaussian noise (AWGN). At the receiver, we apply blanking to mitigate the IN presence and then estimate the optimal output SNR and blanking threshold through optimal search. In all the simulations, the following parameters are used; SNR $=50 \mathrm{~dB}$, $N=4096, \mu_{m c}=10, \mu_{L M C}=0.001, K=1.2, c=0.85$, $d_{5}=1, a_{3}=2$ and $b_{3}=2$. Meanwhile, we confirm that based on the analytical expressions in (20) and (23), all simulated results of the conventional OFDM strongly agree with the analytical results.

Considering the optimal blanking threshold search, it is important to correctly determine the blanking threshold to avoid falsely blanking unintended signals which will severely degrade the output SNR. Consequently, for the uncompanded signal, we recall from the PDF plot in Fig. 1 that the distribution of the signal amplitudes is non-uniform, thus it becomes difficult to correctly determine the correct IN present in the system. In Fig. 4, it becomes that optimal blanking thresholds at different SINR are lower (in other words better estimated) when OFDM signals are companded than when they are not; with ERFC and MC scheme showing the minimum blanking threshold at $p=0.01$.

Furthermore, we increase the probability of IN occurrence in the system by $10 \%$ such that $p=0.1$. The results shown in Fig. 5 demonstrate that companding the OFDM signals increases the probability of correctly blanking the signals. However, the ERFC scheme shows the lowest optimal blanking threshold while HASC is the worst although all companding schemes perform better in terms of optimal blanking threshold than the uncompanded OFDM signal.

Considering the output SNR with respect to the SINR when the probability of IN occurrence is moderate, for example at $p=0.01$. The results shown in Fig. 6 demonstrate that companding OFDM signals improves the output SNR by up to $3.3 \mathrm{~dB}$ gain. Among the companding transform schemes, it can be seen that the MC and ERFC schemes achieve the highest SNR output.

Extending the investigation to powerline channel assumed to be dominated by high IN interference. For example, by

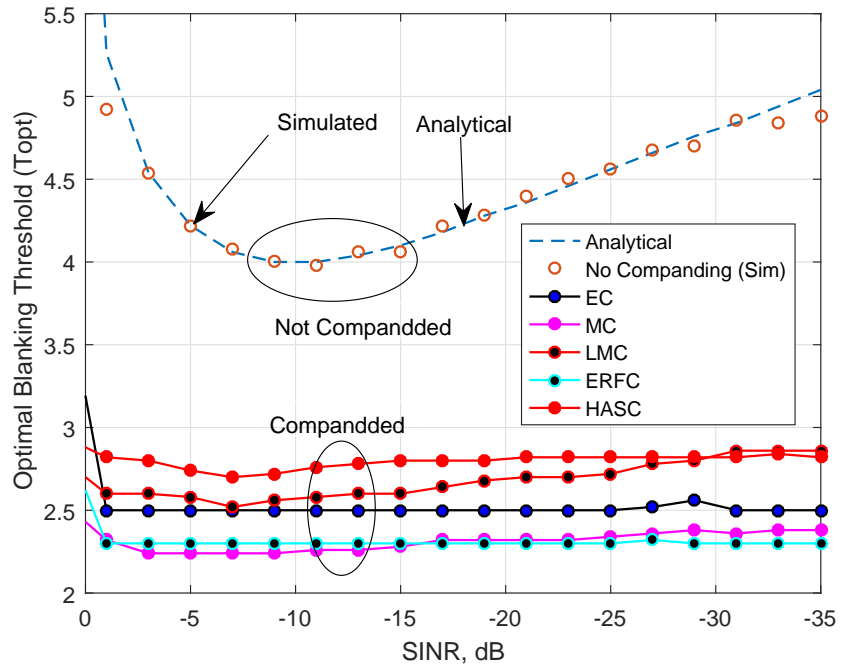

Figure 4. Comparison optimal output SNR results of the OFDM system over impulsive noise channel with AWGN with respect to the SINR; $p=0.01$, $N=4096$

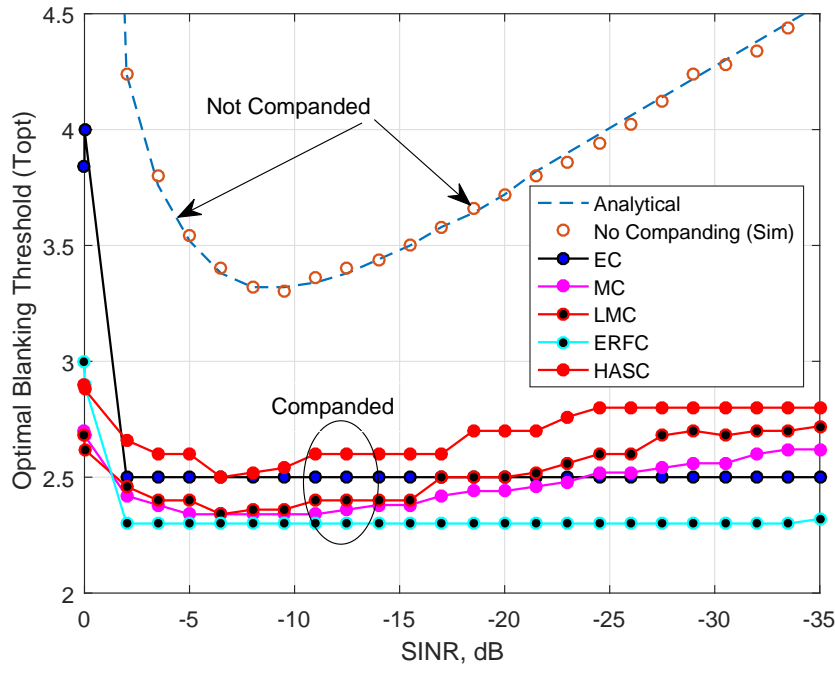

Figure 5. Comparison optimal output SNR results of the OFDM system over impulsive noise channel with AWGN with respect to the SINR; $p=0.1$

increasing the probability of IN occurrence by $10 \%$ from the previous condition so that $p=0.1$, the output SNR is badly degraded compared to the results in Fig. 6. In this case, the output SNR is also enhanced by companding the OFDM signals before transmission. Numerically, by companding the OFDM signals before transmission, we find that the output SNR is increased by $2.4 \mathrm{~dB}$ compared to the uncompanded signal transmission. From our results, the ERFC portends slightly better performance than all other schemes. However, since there is no further parametric improvement/variation possible in using the ERFC scheme, we recommend in the future an investigation of optimal performance of MC and LMC schemes given the dependency on $0<\mu_{\mathrm{MC}}<\infty$. 


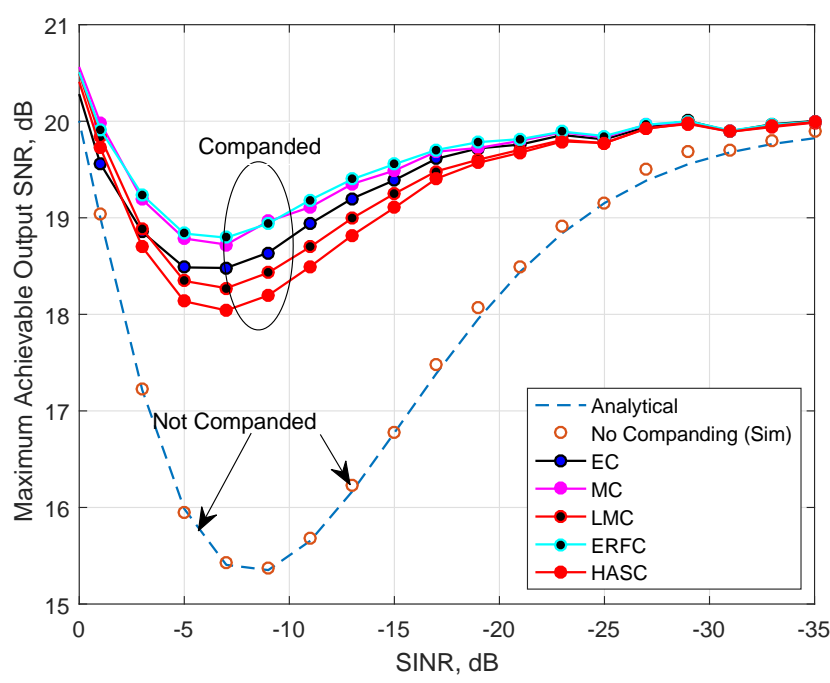

Figure 6. Comparison optimal output SNR results of the OFDM system over impulsive noise channel with AWGN; $p=0.01$

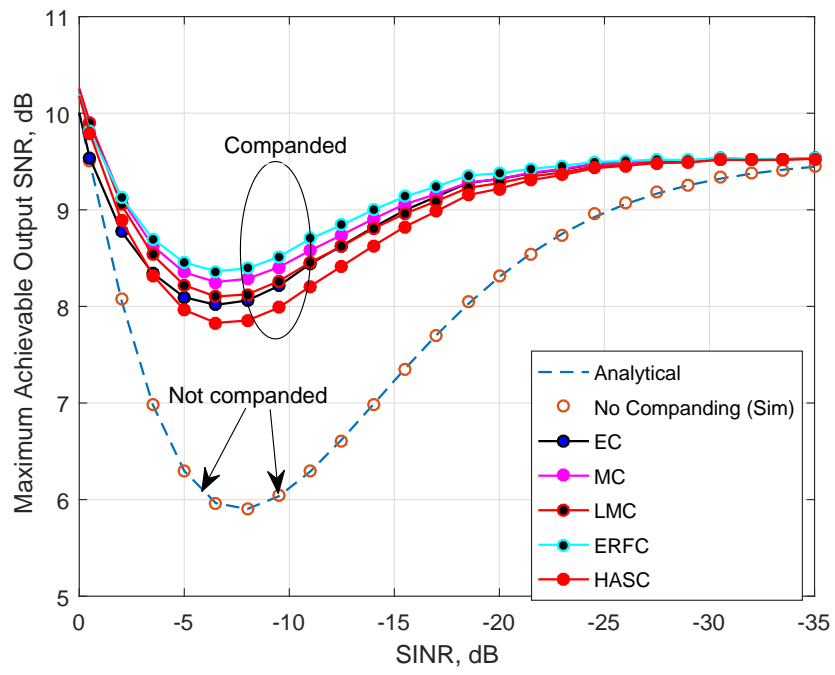

Figure 7. Comparison optimal output SNR results of the OFDM system over impulsive noise channel with AWGN; $p=0.1$

\section{CONCLusions}

In this study, we have presented a method of impulsive noise mitigation by companding OFDM signals. This converts the conventional Rayleigh amplitude distribution to uniform distribution. Five different companding transforms were explored and compared with each other. Then, using a nonlinear optimization search, we found that all companding transforms significantly outperform the conventional OFDM in terms of received SNR and also predict the optimal IN mitigating threshold to exist much below the one presented by the unmodified OFDM amplitude distribution. However, the ERFC scheme shows better SNR output performance among all the schemes. In the future, we suggest the investigation of probability of error reception as degrees of in-band distortions induced into the system by different transforms and their decompanding abilities may impact the likelihood of correctly recovering transmitted signals.

\section{REFERENCES}

[1] Lutz Lampe, Andrea M Tonello, and Theo G Swart. Power Line Communications: Principles, Standards and Applications from multimedia to smart grid. John Wiley \& Sons, second edition, 2016.

[2] Xi Fang, Satyajayant Misra, Guoliang Xue, and Dejun Yang. Smart Grid-The New and Improved Power Grid: A Survey. IEEE Commun. Surveys Tut., 14(4):944-980, 2012.

[3] M. M. Rahman, C. S. Hong, S. Lee, J. Lee, M. A. Razzaque, and J. H. Kim. Medium access control for power line communications: an overview of the IEEE 1901 and ITU-T G.hn standards. IEEE Commun. Mag., 49(6):183-191, Jun. 2011.

[4] Kelvin OO Anoh, James M Noras, Raed A Abd-Alhameed, Steve MR Jones, and Konstantinos N Voudouris. A new approach for designing orthogonal wavelets for multicarrier applications. AEU-Intl. J. Electron. Commun., 68(7):616-622, Jul. 2014.

[5] Kelvin OO Anoh, Trust T Mapoka, Raed A Abd-Alhameed, Ogechukuka Ochonogor, and Steve MR Jones. On the Application of Raised-Cosine Wavelets for Multicarrier Systems Design. IRECAP, 4(4):143-150, Aug. 2014.

[6] Xianbin Wang, T. T. Tjhung, and C. S. Ng. Reduction of peak-toaverage power ratio of OFDM system using a companding technique. IEEE Trans. Broadcast., 45(3):303-307, Sept. 1999.

[7] K. Anoh, C. Tanriover, and B. Adebisi. On the Optimization of Iterative Clipping and Filtering for PAPR Reduction in OFDM Systems. IEEE Access, PP(99):1-1, 2017.

[8] Kelvin Anoh, Raed Abd-Alhameed, Yousef Dama, Steve Jones, Prashant Pillai, and K Voudouris. An investigation of PMEPR of WPT-OFDM and OFDM multicarrier systems. J. Commun. Netw., 3(3):45-52, Jul. 2013.

[9] K. M. Rabie and E. Alsusa. Efficient SLM based impulsive noise reduction in powerline OFDM communication systems. In 2013 IEEE GLOBECOM, pages 2915-2920, Dec. 2013.

[10] K. M. Rabie, E. Alsusa, A. D. Familua, and L. Cheng. Constant envelope OFDM transmission over impulsive noise power-line communication channels. In 2015 IEEE ISPLC, pages 13-18, Mar. 2015.

[11] S. V. Zhidkov. Analysis and comparison of several simple impulsive noise mitigation schemes for OFDM receivers. IEEE Trans. Commun., 56(1):5-9, Jan. 2008.

[12] Jun Hou, Jianhua Ge, Dewei Zhai, and Jing Li. Peak-to-average power ratio reduction of OFDM signals with nonlinear companding scheme. IEEE Transac. Broadcast., 56(2):258-262, 2010.

[13] Tao Jiang, Weidong Xiang, Paul C Richardson, Daiming Qu, and Guangxi Zhu. On the nonlinear companding transform for reduction in PAPR of MCM signals. IEEE Trans. Wireless Commun., 6(6):20172021, Jun. 2007.

[14] O. Gazi. A new companding technique for PAPR reduction in OFDM communication systems. In 3rd ICUMT, pages 1-5, Oct. 2011.

[15] Rola Almahainy, Nazar Ali, Saleh Al-Araji, and Mohammed Ismail. Impulsive noise mitigation for $\mu$-law companded OFDM-based communication system. In IEEE ICECS, pages 264-267, Dec. 2015.

[16] Y. Wang, L. h. Wang, J. h. Ge, and B. Ai. Nonlinear companding transform technique for reducing PAPR of OFDM signals. IEEE Trans. Consum. Electron., 58(3):752-757, Aug. 2012.

[17] Tao Jiang, Yang Yang, and Yong-Hua Song. Exponential companding technique for PAPR reduction in OFDM systems. IEEE Trans. Broadcast., 51(2):244-248, Jun. 2005.

[18] Jing Lin, Marcel Nassar, and Brian L Evans. Impulsive Noise Mitigation in Powerline Communications Using Sparse Bayesian Learning. IEEE J. Sel. Areas Commun., 31(7):1172-1183, Mar. 2013.

[19] Paolo Banelli. Non-linear transformations of Gaussians and Gaussianmixtures with implications on estimation and information theory. arXiv preprint arXiv:1111.5950v3, May 2013.

[20] Ryota Yoshizawa and Hideki Ochiai. Effect of Clipping and Filtering with Distortionless PAPR Reduction for OFDM Systems. In 2015 IEEE 82nd VTC Fall, pages 1-5, Sept. 2015.

[21] Bamidele Adebisi, Khaled Maaiuf Rabie, Augustine Ikpehai, Cinna Soltanpur, and Andrew Wells. Vector ofdm transmission over nongaussian power line communication channels. IEEE Syst. J., 2017. 